\title{
Validación de un instrumento para medir la vulnerabilidad en relación con la capacidad de respuesta de la comunidad ante desastres
}

\section{Validation of an instrument to measure the vulnerability in relation to the community's response capacity front of disasters}

\author{
Jorge Norvey Álvarez-Ríos ${ }^{1}$ \\ Paula Andrea Aristizábal-Vélez² \\ Diana María Torres-Pavas ${ }^{3}$ \\ Valentina Jurado-Alzate ${ }^{4}$ \\ Universidad Autónoma de Manizales, Caldas, Colombia
}

\begin{abstract}
Resumen
La validación de instrumentos es uno de los procedimientos indispensables para garantizar la confiabilidad y validez en la medición de variables. En gestión del riesgo de desastres existe la necesidad de medir las amenazas y el nivel de vulnerabilidad, según diferentes autores. Objetivo: validar un instrumento para medir la vulnerabilidad en relación con la capacidad de respuesta de la comunidad ante desastres. Metodología: se diseñó una encuesta semiestructurada, la cual fue validada por un panel de expertos a través de la calificación de indicadores. Se aplicó la prueba Alfa de Cronbach. Resultados: el instrumento consta de 52 preguntas, las cuales fueron validadas por expertos, donde la prueba de validez en cuanto a suficiencia, coherencia, claridad y relevancia dio mayor a 0,9 , esto indica que existe concordancia entre los evaluadores.
\end{abstract}

Palabras Clave: Vulnerabilidad ante desastres, estudios de validación, validez de las pruebas y confiabilidad. (DeCS Server - List Terms)

1 Magister en Educación. Docencia. Antigua estación del ferrocarril Universidad Autónoma de Manizales. Caldas, Colombia. Correo electrónico: Jnalvarez@autonoma.edu.co

2 Magister en Salud Pública. Docencia. Antigua estación del ferrocarril Universidad Autónoma de Manizales. Caldas, Colombia. Correo electrónico: paulaaristizabal@autonoma.edu.co

3 Magister en Salud Pública. Docencia. Antigua estación del ferrocarril Universidad Autónoma de Manizales. Caldas, Colombia. Correo electrónico: dtorres@autonoma.edu.co

4 Estudiante Tecnología en Atención Prehospitalaria. Antigua estación del ferrocarril Universidad Autónoma de Manizales. Caldas, Colombia. Correo electrónico: Valentina.juradoa@autonoma.edu.co 
Jorge Norvey Álvarez-Ríos, Paula Andrea Aristizábal-Vélez, Diana María Torres-Pavas, Valentina Jurado-Alzate. Validation of an instrument to measure the vulnerability in relation to the community's response capacity front of disasters

\begin{abstract}
The validation of instruments is one of the indispensable procedures to guarantee the reliability and validity in the measurement of variables. In disaster risk management, there is a need to measure the threats and the level of vulnerability according to the different authors. Objective: To validate an instrument to measure vulnerability in relation to the response capacity of the community front of disasters. Methodology: a semi-structured survey was designed, which was validated by an expert panel through the qualification of the indicators. It applies to the Alpha Cronbach test. Results: the instrument consists of 52 questions, which were validated by experts where the validity test in terms of sufficiency, coherence, clarity and relevance gave more than 0.9 this indicates that there is agreement between the evaluators.
\end{abstract}

Keywords: Vulnerability front of disasters, validation studies, validity of the tests and reliability. (DeCS Server - List Terms).

\title{
Introducción
}

En la investigación con enfoque cuantitativo, el instrumento se constituye en un elemento esencial para la recolección de información; este, permite medir las variables, las cuales surgen de los objetivos y el marco teórico. Para lograr lo anterior, estadísticamente se recomienda que las preguntas y, en general, el instrumento deba contar con validez de constructo, de confiabilidad y de contenido. Lo anterior, garantiza que los instrumentos empleados en investigaciones con enfoque cuantitativo cuenten con validez, de tal forma, que la información obtenida, realmente, sea veraz y coherente con lo medido (Pérez \& Martínez, 2008).

En la gestión del riesgo en desastres es necesario conocer las amenazas, la vulnerabilidad y con esto el riesgo. La amenaza, es definida como la posibilidad de ocurrencia de un fenómeno y que pueda causar daño a una comunidad. La vulnerabilidad, se entiende como la susceptibilidad de las personas y/o las comunidades ante las amenazas. Y el riesgo, es la relación constante entre la amenaza y la vulnerabilidad. Para conocer el riesgo, es necesario realizar una medición de la probabilidad y magnitud de las amenazas; pero. además, es fundamental identificar el nivel de vulnerabilidad de las comunidades. Esto permite centrar las intervenciones desde las prioridades, de una manera objetiva y coherente, procurando disminuir la vulnerabilidad (Rojas Vilches \& Martínez Reyes, 2011).

Por consiguiente, es fundamental contemplar la necesidad del uso de instrumentos para la medición no solo de las amenazas, sino también de la vulnerabilidad de las comunidades. Es así, como el Marco de Acción de Sendai para la reducción del riesgo de desastres, establece la necesidad del 
Jorge Norvey Álvarez-Ríos, Paula Andrea Aristizábal-Vélez, Diana María Torres-Pavas, Valentina Jurado-Alzate.

Validación de un instrumento para medir la vulnerabilidad en relación con la capacidad

de respuesta de la comunidad ante desastres

conocimiento del índice de amenazas y la vulnerabilidad de las regiones, para tomar medidas a través de las políticas públicas (Casar, 2015). De este modo, se evidencia la necesidad de incluir instrumentos validados, que permitan la medición de la vulnerabilidad de una manera asertiva según los objetivos planteados.

Algunos autores han desarrollado instrumentos que permiten la medición de la vulnerabilidad de las comunidades. Bohórquez (2013), desarrolló en su estudio denominado "evaluación de la vulnerabilidad social ante amenazas naturales en Manzanillo (Colima)", un instrumento para medir la vulnerabilidad a través del uso del método ACP (Análisis de Componente Principales) (Thomas Bohórquez, 2013).

De igual forma, Chardon en su estudio "un enfoque geográfico de la vulnerabilidad en zonas urbanas expuestas a amenazas naturales", plantea un instrumento para medir la vulnerabilidad de las comunidades a través del uso de ACP (Chardon, 2002). Ambos estudios, cuentan con instrumentos empleados para la medición de variables que dan respuesta al reconocimiento de la vulnerabilidad; estudios como estos, motivan al investigador a realizar procedimientos que, mediante el método científico, permitan obtener validez y confiabilidad de los instrumentos.

De acuerdo con la búsqueda en bases de datos, se identifican pocos estudios que den cuenta de un instrumento para medir la vulnerabilidad ante desastres por parte de las comunidades. Es así, como surgió el presente proyecto con el fin de validar un instrumento a través de métodos científicos que permitiera medir la vulnerabilidad en relación con la capacidad de respuesta de las comunidades ante desastres.

\section{Materiales y Métodos}

Este fue un estudio cuantitativo enfocado a obtener la validez de contenido y confiabilidad de un instrumento que se basa en una encuesta semiestructurada, la cual se aplicará a domicilio por un encuestador debidamente entrenado.

Los criterios de inclusión en el momento de responder la encuesta son: a) La persona encuestada debe ser mayor a de edad (mayor a 18 años); b) La familia a la cual representa, debe tener un año o más de antigüedad de vivir en el barrio; y c) La persona que responde debe ser parte del núcleo familiar. Se considera como criterio de exclusión: la persona que responda 
la encuesta no puede tener una condición clínica o de discapacidad cognitiva. Finalmente, dentro del entrenamiento se indicará al encuestador no generar preguntas adicionales que impliquen un sesgo en la investigación o que promueva alguna respuesta en particular. La encuesta cuenta con ocho componentes los cuales se describirán a continuación.

1. Información de la persona encuestada (total preguntas: 4). En este componente se abordarán variables que permiten establecer una relación entre características sociodemográficas e información como: sexo, ocupación, edad y máximo nivel de escolaridad. Algunos estudios, han demostrado diferencias significativas en la percepción del riesgo entre género, ocupación y nivel de escolaridad. (Chávez \& Ballesteros, 2002) (Cid Ortiz, Castro Correa, \& Rugiero de Souza, 2012)

2. Características sociodemográficas (total preguntas: 7). Una vez detallada la información de la persona a la cual se le está aplicando la encuesta, continúan las variables sociodemográficas: dirección, barrio, número de personas que habitan la vivienda (por edad y condiciones de discapacidad), antigüedad en el barrio, motivo para vivir en el barrio, organizaciones a las cuales pertenece algún integrante de la familia y máximo nivel de escolaridad de algún integrante de la familia. Algunos autores, establecen que la vulnerabilidad debe incluir parámetros sociodemográficos. (Sánchez-González \& Egea-Jiménez, 2011)

3. Características económicas (total preguntas: 2). En este componente se incluyen variables, tales como ingresos mensuales familiares promedio y estrato socioeconómico. Esta medición posibilitará identificar la capacidad de recuperación ante un evento adverso de la familia. Algunos autores, establecen que el factor económico influye considerablemente en la recuperación, tanto del país como de la familia, una vez ocurra el evento. (Bohórquez \& Enrique, 2011)

4. Características de la vivienda (total preguntas: 13). En este componente se evalúan las variables: tenencia de la vivienda, tipo de vivienda, número de niveles, tipo de construcción, área aproximada en metros cuadrados, mantenimiento de la vivienda, incorporación de normas de sismo resistencia, destinación del uso del suelo, servicios en el hogar y servicios colectivos en el barrio. Este tipo de variables permitirá identificar la vulnerabilidad en relación con la vivienda 
que se habita. En este aspecto, algunos estudios han mostrado evidencias de la relación entre el tipo de vivienda y el impacto de los desastres, la mala calidad de las viviendas aumenta la vulnerabilidad. (Mardones \& Vidal, 2001)

5. Amenazas de origen natural (total preguntas: 5). En este caso, se pregunta sobre el conocimiento de amenazas que puedan afectar la vivienda, además de la calificación de la amenaza (posible, probable e inminente según su probabilidad de ocurrencia), antecedentes de amenazas ocurridas en el barrio. (Medina \& Adolfo, 2013)

6. Amenazas de origen antrópico (total preguntas: 5). De igual forma, como ocurrió en el anterior punto, es necesaria la calificación de la amenaza, abordar las variables de conocimiento, calificación según probabilidad de ocurrencia y antecedentes en el barrio. (Medina \& Adolfo, 2013)

7. Organización ante las amenazas (total preguntas: 3 ). En este aspecto, se incluyen variables, tales como: plan familiar de gestión del riesgo de desastres, plan de evacuación y puntos de encuentro. El pensar la gestión del riesgo desde la familia es uno de los pilares fundamentales y se constituye en un factor que disminuye la vulnerabilidad. (Soares \& Murillo-Licea, 2013)

8. Preparación ante las amenazas (total preguntas: 12). Una vez identificadas las amenazas y la organización ante el riesgo de desastres, es indispensable evaluar la preparación, para esto se incluyen variables como: formación en gestión del riesgo, conocimiento del plan de gestión del riesgo de la ciudad, conocimiento e implementación de alarmas de evacuación, dotación de botiquines, inventario de extintores y capacitación recibida en relación con primeros auxilios y manejo de extintores. Este componente permite identificar la preparación de la comunidad a partir de sus capacidades instaladas, contando con dotación básica y conocimiento sobre gestión del riesgo de desastres.

Teniendo en cuenta lo anterior, se puede llegar a medir la vulnerabilidad en relación con la capacidad de respuesta, desde el concepto se mide en términos de consecuencias potenciales (cantidad y cualidad de los efectos). Es importante recordar que el riesgo se establece con la relación existente entre amenaza y vulnerabilidad. (Aguirre, 2004). 
Validez de contenido a través de juicios o panel de expertos. Este método es definido como una opinión informada de personas con trayectoria en el tema, que son identificados por otros como cualificados para dar información, evidencia, juicios y valoraciones. (Nova, Mosqueda, \& Tobón, 2016) Para la validación del instrumento, se tuvo apoyo de cinco expertos en gestión del riesgo de desastres con formación de posgrado (especialización o maestría), con experiencia en proyectos de investigación y validación de instrumentos. Los criterios establecidos para la validación fueron:

Tabla 1. Criterios de calificación tomado de (Pérez \& Martínez, 2008)

\begin{tabular}{|c|c|c|}
\hline CATEGORIA & CALIFICACIÓN & INDICADOR \\
\hline \multirow{4}{*}{$\begin{array}{l}\text { SUFICIENCIA } \\
\text { Los ítems que pertenecen } \\
\text { a una misma dimensión } \\
\text { bastan para obtener la } \\
\text { medición de ésta. }\end{array}$} & 1. No cumple con el criterio & Los ítems no son suficientes para medir la dimensión \\
\hline & 2. Bajo Nivel & $\begin{array}{l}\text { Los ítems miden algún aspecto de la dimensión, } \\
\text { pero no corresponden con la dimensión total }\end{array}$ \\
\hline & 3. Moderado nivel & $\begin{array}{l}\text { Se deben incrementar algunos ítems para poder } \\
\text { evaluar la dimensión completamente. }\end{array}$ \\
\hline & 4. Alto nivel & Los ítems son suficientes \\
\hline \multirow{4}{*}{$\begin{array}{l}\text { CLARIDAD } \\
\text { El ítem se comprende } \\
\text { fácilmente, es decir, su } \\
\text { sintáctica y semántica } \\
\text { son adecuadas. }\end{array}$} & 1. No cumple con el criterio & El ítem no es claro \\
\hline & 2. Bajo Nivel & $\begin{array}{l}\text { El ítem requiere bastantes modificaciones o una } \\
\text { modificación muy grande en el uso de las palabras } \\
\text { de acuerdo con su significado o por la ordenación } \\
\text { de las mismas }\end{array}$ \\
\hline & 3. Moderado nivel & $\begin{array}{l}\text { Se requiere una modificación muy específica de } \\
\text { algunos de los términos del ítem. }\end{array}$ \\
\hline & 4. Alto nivel & El ítem es claro, tiene semántica y sintaxis adecuada. \\
\hline \multirow{4}{*}{$\begin{array}{l}\text { COHERENCIA } \\
\text { El ítem tiene relación } \\
\text { lógica con la dimensión } \\
\text { o indicador que está } \\
\text { midiendo. }\end{array}$} & 1. No cumple con el criterio & El ítem no tiene relación lógica con la dimensión \\
\hline & 2. Bajo Nivel & El ítem tiene una relación tangencial con la dimensión. \\
\hline & 3. Moderado nivel & $\begin{array}{l}\text { El ítem tiene una relación moderada con la } \\
\text { dimensión que está midiendo. }\end{array}$ \\
\hline & 4. Alto nivel & $\begin{array}{l}\text { El ítem se encuentra completamente relacionado } \\
\text { con la dimensión que está midiendo }\end{array}$ \\
\hline \multirow{4}{*}{$\begin{array}{l}\text { RELEVANCIA } \\
\text { El ítem es esencial o } \\
\text { importante, es decir debe } \\
\text { ser incluido. }\end{array}$} & 1. No cumple con el criterio & $\begin{array}{l}\text { El ítem puede ser eliminado sin que se vea } \\
\text { afectada la medición de la dimensión }\end{array}$ \\
\hline & 2. Bajo Nivel & $\begin{array}{l}\text { El ítem tiene alguna relevancia, pero otro ítem } \\
\text { puede estar incluyendo lo que mide éste }\end{array}$ \\
\hline & 3. Moderado nivel & El ítem es relativamente importante \\
\hline & 4. Alto nivel & El ítem es muy relevante y debe ser incluido \\
\hline
\end{tabular}

A cada uno de los expertos se le hizo entrega de: objetivo del estudio, parametrización de variables, objetivo de la prueba, pesos y diferencias en las dimensiones y la encuesta previamente diseñada. 
Jorge Norvey Álvarez-Ríos, Paula Andrea Aristizábal-Vélez, Diana María Torres-Pavas, Valentina Jurado-Alzate.

Validación de un instrumento para medir la vulnerabilidad en relación con la capacidad

de respuesta de la comunidad ante desastres

De igual forma, se aplicó prueba piloto para medir la validez de constructo. Esta prueba fue aplicada de manera aleatoria en población que cumplía con los criterios de inclusión y exclusión estimados en el diseño. El objetivo principal fue evaluar aspectos técnicos, mejorar y corregir todas las deficiencias en el instrumento.

La prueba aplicada fue Alfa de Cronbach, la cual estableció el nivel de concordancia entre un conjunto de rangos. En este caso los rangos establecidos fueron de 1 a 4, donde 1 no cumple el nivel, 2 bajo nivel, 3 moderado nivel y 4 alto nivel. Estos criterios aplicaban para las categorías de suficiencia, claridad, coherencia y relevancia. Se aplicó esta prueba, una vez identificados y cambiados los ítems sugeridos por el panel de expertos. Si el valor es mayor a 0.5 (ideal cercano al valor de 1) se podía concluir que hay acuerdo entre los evaluadores. Para realizar el análisis estadístico, se utilizó el paquete estadístico SPSS versión 24, donde la Universidad Autónoma de Manizales cuenta con licencia adquirida.

\section{Resultados y Discusión}

En los resultados de la validación del instrumento se analizaron los ítems y/o variables que tienen diferencias significativas en su valoración. En total el instrumento está compuesto por 52 preguntas. A continuación, se presenta el análisis cuantitativo, cualitativo y prueba piloto.

\section{Análisis cuantitativo}

Tabla 2. Alfa Cronbach

\begin{tabular}{|l|c|c|c|c|}
\hline & SUFICIENCIA & COHERENCIA & RELEVANCIA & CLARIDAD \\
\hline SUFICIENCIA & 1,000 & 0,990 & 0,980 & 0,980 \\
\hline COHERENCIA & 0,990 & 1,000 & 0,990 & 0,990 \\
\hline RELEVANCIA & 0,980 & 0,990 & 1,000 & 1,000 \\
\hline CLARIDAD & 0,980 & 0,990 & 1,000 & 1,000 \\
\hline
\end{tabular}

Los resultados obtenidos en cuanto al grado de concordancia externa, a través del juicio de expertos a cada ítem. Una vez aplicada la prueba de Alfa Cronbach, se identifica que el nivel es significativo entre rangos, se evidencia que la suficiencia, coherencia, relevancia y claridad superan el valor de $0,5 \mathrm{y}$ está muy cercano al valor de 1. Lo anterior, permite identificar que los indicadores son adecuados, aunque son susceptibles de mejora en aquellos ítems 
en los cuales algún juez los calificó con valor de 1 en una escala de 1 a 4 . En conclusión, los ítems y el instrumento cuentan con validez y confiabilidad.

Se evidencia una concordancia entre los jueces, pero es necesario analizar de manera específica cada ítem. Este procedimiento, permitirá excluir del instrumento aquellas variables con calificación con una media baja. Este análisis, de igual forma, depende del análisis cualitativo brindado por los jueces.

Tabla 3. Media en cada ítem

\begin{tabular}{|c|c|c|c|c|c|}
\hline & & \multicolumn{4}{|c|}{ MEDIA } \\
\hline DIMENSIÓN & ITEM & Suficiencia & Coherencia & Relevancia & Claridad \\
\hline \multirow{7}{*}{$\begin{array}{l}\text { 1. CARACTERÍSTICAS } \\
\text { SOCIODEMOGRÁFICAS }\end{array}$} & Ítem 1.1. & 4,00 & 4,00 & 4,00 & 4,00 \\
\hline & Ítem 1.2. & 3,67 & 3,67 & 3,67 & 3,67 \\
\hline & Ítem 1.3. & 3,33 & 3,33 & 3,33 & 3,33 \\
\hline & Ítem 1.4. & 4,00 & 4,00 & 4,00 & 4,00 \\
\hline & Ítem 1.5. & 4,00 & 4,00 & 4,00 & 4,00 \\
\hline & Ítem 1.6. & 3,67 & 3,67 & 3,67 & 3,67 \\
\hline & Ítem 1.7. & 4,00 & 4,00 & 4,00 & 4,00 \\
\hline \multirow{4}{*}{$\begin{array}{l}\text { 2. INFORMACIÓN } \\
\text { DE LA PERSONA } \\
\text { ENCUESTADA }\end{array}$} & Ítem 2.1. & 4,00 & 4,00 & 4,00 & 4,00 \\
\hline & Ítem 2.2. & 4,00 & 4,00 & 4,00 & 4,00 \\
\hline & Ítem 2.3. & 4,00 & 4,00 & 4,00 & 4,00 \\
\hline & Ítem 2.4. & 4,00 & 4,00 & 4,00 & 4,00 \\
\hline \multirow{2}{*}{$\begin{array}{l}\text { 3. CARACTERÍSTICAS } \\
\text { ECONÓMICAS }\end{array}$} & Ítem 3.1. & 4,00 & 4,00 & 4,00 & 4,00 \\
\hline & Ítem 3.2. & 4,00 & 4,00 & 4,00 & 4,00 \\
\hline \multirow{13}{*}{$\begin{array}{l}\text { 4. CARACTERÍSTICAS } \\
\text { DE LA VIVIENDA }\end{array}$} & Ítem 4.1. & 4,00 & 4,00 & 4,00 & 4,00 \\
\hline & Ítem 4.2. & 4,00 & 4,00 & 4,00 & 4,00 \\
\hline & Ítem 4.3. & 4,00 & 4,00 & 4,00 & 4,00 \\
\hline & Ítem 4.4. & 4,00 & 4,00 & 4,00 & 4,00 \\
\hline & Ítem 4.5. & 3,67 & 3,67 & 3,67 & 3,67 \\
\hline & Ítem 4.6. & 3,67 & 3,67 & 3,67 & 3,67 \\
\hline & Ítem 4.7. & 4,00 & 4,00 & 4,00 & 4,00 \\
\hline & Ítem 4.8. & 4,00 & 4,00 & 4,00 & 4,00 \\
\hline & Ítem 4.9. & 3,33 & 3,33 & 3,33 & 3,33 \\
\hline & Ítem 4.10. & 3,67 & 3,67 & 3,67 & 3,67 \\
\hline & Ítem 4.11. & 4,00 & 4,00 & 4,00 & 4,00 \\
\hline & Ítem 4.12. & 4,00 & 4,00 & 4,00 & 4,00 \\
\hline & Ítem 4.13. & 3,67 & 3,67 & 3,67 & 3,67 \\
\hline
\end{tabular}


Jorge Norvey Álvarez-Ríos, Paula Andrea Aristizábal-Vélez, Diana María Torres-Pavas, Valentina Jurado-Alzate. Validación de un instrumento para medir la vulnerabilidad en relación con la capacidad de respuesta de la comunidad ante desastres

\begin{tabular}{|l|c|c|c|c|c|}
\hline & & \multicolumn{4}{|c|}{ MEDIA } \\
\hline \multicolumn{1}{|c|}{ DIMENSIÓN } & ITEM & Suficiencia & Coherencia & Relevancia & Claridad \\
\hline 5. AMENAZAS & Ítem 5.1. & 3,00 & 3,00 & 3,00 & 3,00 \\
\cline { 2 - 6 } & Ítem 5.2. & 3,33 & 3,33 & 3,33 & 3,33 \\
\cline { 2 - 6 } & Ítem 5.3. & 4,00 & 4,00 & 4,00 & 4,00 \\
\cline { 2 - 6 } & Ítem 5.4. & 4,00 & 4,00 & 4,00 & 4,00 \\
\cline { 2 - 6 } & Ítem 5.5. & 4,00 & 4,00 & 4,00 & 4,00 \\
\hline \multirow{4}{*}{ 6. AMENALES } & Ítem 6.1. & 3,67 & 3,67 & 3,67 & 3,67 \\
\cline { 2 - 6 } & Ítem 6.2. & 3,67 & 3,67 & 3,67 & 3,67 \\
\cline { 2 - 6 } & Ítem 6.3. & 3,67 & 3,67 & 3,67 & 3,67 \\
\cline { 2 - 6 } & Ítem 6.4. & 4,00 & 4,00 & 4,00 & 4,00 \\
\cline { 2 - 6 } & Ítem 6.5. & 4,00 & 4,00 & 4,00 & 4,00 \\
\hline 7. ORGANIZACIÓN & Ítem 7.1. & 4,00 & 4,00 & 4,00 & 4,00 \\
\cline { 2 - 6 } ANTE LAS AMENAZAS & Ítem 7.2. & 4,00 & 4,00 & 4,00 & 4,00 \\
\cline { 2 - 6 } & Ítem 7.3. & 3,00 & 3,00 & 3,00 & 3,00 \\
\cline { 2 - 6 } & Ítem 7.4. & 4,00 & 4,00 & 4,00 & 4,00 \\
\hline 8. PREPARACIÓN ANTE & Ítem 8.1. & 3,67 & 3,67 & 3,67 & 3,67 \\
\cline { 2 - 6 } LAS AMENAZAS & Ítem 8.2. & 3,33 & 3,33 & 3,33 & 3,33 \\
\cline { 2 - 6 } & Ítem 8.3. & 3,67 & 3,67 & 3,67 & 3,67 \\
\cline { 2 - 6 } & Ítem 8.4. & 3,67 & 3,67 & 3,67 & 3,67 \\
\cline { 2 - 6 } & Ítem 8.5. & 4,00 & 4,00 & 4,00 & 4,00 \\
\cline { 2 - 6 } & Ítem 8.6. & 4,00 & 4,00 & 4,00 & 4,00 \\
\cline { 2 - 6 } & Ítem 8.7. & 4,00 & 4,00 & 4,00 & 4,00 \\
\cline { 2 - 6 } & Ítem 8.8. & 4,00 & 4,00 & 4,00 & 4,00 \\
\cline { 2 - 6 } & Ítem 8.9. & 4,00 & 4,00 & 4,00 & 4,00 \\
\cline { 2 - 6 } & Ítem 8.10. & 3,00 & 3,00 & 3,00 & 3,00 \\
\cline { 2 - 6 } & Ítem 8.11. & 3,33 & 3,33 & 3,33 & 3,33 \\
\cline { 2 - 6 } & Ítem 8.12. & 4,00 & 4,00 & 4,00 & 4,00 \\
\hline
\end{tabular}

En un análisis cuantitativo de las evaluaciones obtenidas por los expertos, de cada criterio por ítem, es posible determinar el grado de fiabilidad en cada uno de ellos. Es decir, a partir de la valoración brindada en un rango de 1 a 4, en función de la suficiencia, claridad, coherencia y relevancia. De los 52 ítems, 31 (59\%) fueron calificados con un valor de 4, es decir de "alto nivel" y los 21 (41\%) restantes con un promedio entre 3 y 4 , una valoración que equivale a "moderado nivel". La pregunta con más baja calificación fueron las relacionadas con: rutas de evacuación en la vivienda (ítem 7.3.), conocimiento de la amenaza natural (ítem 5.1.) y formación en RCP (ítem 8.10) (Promedio=3). Este análisis, permite establecer los ítems que se deben fortalecer o definitivamente no incluirlos. 


\section{Análisis cualitativo}

A parte de la valoración por ítem, se proporcionó un espacio para las observaciones por parte del experto. Este procedimiento, permitió evidenciar sugerencias y recomendaciones pertinentes para el análisis cualitativo. A continuación, se indican las observaciones.

\section{- Suficiencia}

En la evaluación por parte del panel de expertos, se le dio un valor menor en la pregunta relacionada con "las rutas de evacuación", está asociada a la recomendación, que las viviendas no deberían contar con rutas de evacuación, solo aquellas edificaciones enmarcadas como edificios y conjuntos residenciales. Se puede especificar en este aspecto la posibilidad de respuesta "no aplica".

Al realizar un análisis comparado con los referentes teóricos, se identifica la necesidad de ubicar el ítem 7.3, el cual está sustentado en la necesidad de contar con rutas de evacuación externas a la vivienda, no solo dentro de ella. Se han identificado algunos estudios, en los cuales se establecen las rutas de evacuación sobre fenómenos específicos, como tsunamis y deslizamientos, entre otros ( $\mathrm{P} \& \mathrm{H}, 2011)$. Además, se han identificado esfuerzos para garantizar las rutas de evacuación a través de sistemas de georreferenciación y mapas, para garantizar la disminución de los efectos de los desastres (Montenegro-Romero \& Peña-Cortés, 2010).

Una de las observaciones planteadas por los expertos, es la necesidad de incluir si algún miembro de la familia es integrante de una brigada de emergencias empresarial, grupo scout o grupo pastoral. Lo anterior, se puede entender como escenarios que favorecen el desarrollo de habilidades para la prevención y atención de las emergencias y los desastres. Por la dinámica en el campo de la gestión del riesgo de desastre, se identifican avances en la formación de grupos poblacionales para afrontar situaciones de emergencias y desastres.

Es el campo escolar, donde los jóvenes pueden tener una formación básica sobre gestión del riesgo (Fajardo-Maldonado et al., 2009). De igual forma, la formación de brigadas de emergencia se ha identificado como elemento esencial en la respuesta prehospitalaria de eventos con múltiples víctimas (Leonardo Ristori, 2011). 
Jorge Norvey Álvarez-Ríos, Paula Andrea Aristizábal-Vélez, Diana María Torres-Pavas, Valentina Jurado-Alzate.

Validación de un instrumento para medir la vulnerabilidad en relación con la capacidad

de respuesta de la comunidad ante desastres

Algunos expertos, mencionan que es necesario incluir otros elementos, entre ellos: si la persona que responde es cabeza de hogar, si cuenta con afiliación a seguridad social y afiliación a pensión. En este aspecto es importante resaltar, que en Colombia la afiliación al sistema general de seguridad y salud es obligatoria, la que se hace a través de las entidades promotoras de salud (EPS), las cuales ofrecen el plan obligatorio de salud (POS) o el POS S para el régimen subsidiado (Guerrero, Gallego, BecerrilMontekio, \& Vásquez, 2011; Vargas J \& Molina M, 2009).

\section{- Coherencia}

Dentro de las observaciones pertinentes a la suficiencia, se destaca la necesidad de poner la opción de barrios, según normatividad legal vigente para las ciudades, en la cual se establece la distribución geográfica y los límites entre barrios. Existen opciones de respuesta que no son útiles para el propósito del instrumento, entre ellas, el incluir en la caracterización si existen canchas o escenarios deportivos. Frente a este aspecto, se realiza una revisión en las bases de datos y no se evidencia sustento para incluir los escenarios deportivos. Si bien, se han utilizado como puntos de encuentro, no siempre son escenarios idóneos y accesibles para este propósito. Los escenarios deportivos se han caracterizado como lugares que favorecen la promoción de la salud, el ocio y la lúdica en niños, pero no existe soporte científico de que todos sirvan para la atención de desastres (Lavin Fueyo \& Berra, 2015).

Por recomendación y evidencia, se excluye de la opción de respuesta parques y escenarios deportivos. Se deja este aspecto, para un análisis técnico que deberá desarrollar las entidades gubernamentales a través de los planes comunitarios de gestión del riesgo de desastres.

\section{- Relevancia}

En las observaciones, los expertos hacen énfasis en delimitar el nivel de la formación solo en formación técnica, tecnológica y profesional. Lo anterior, de manera diferente como fue planteado en el instrumento, en el cual se segmenta por niveles de formación (Básica primaria, básica secundaria, técnico, profesional, especialización, maestría y doctorado). No existen referentes que permita evidenciar la relación entre conocimiento del riesgo y el nivel de formación. Existe un estudio, el cual informa de 
manera superficial sobre el abordaje de la gestión del riesgo desde las diferentes disciplinas (Echemendía Tocabens, 2011).

\section{- Claridad}

En este aspecto, los expertos mencionan que es necesario aclarar a quien va dirigido el instrumento. De igual forma, hacen énfasis a la permanencia en el hogar, debido a que existen personas que pueden estar habitando la vivienda de manera transitoria. Por otro lado, hacen énfasis en no ubicar siglas que pueda generar confusiones en el encuestador.

Los expertos mencionan, la necesidad de analizar que existen viviendas que brindan servicios de alojamiento por habitaciones. Es decir, un estudiante puede arrendar una habitación y no necesariamente ser de la familia. Este análisis es útil en la discusión sobre el tipo de vivienda, a la cual se le debe incluir aparta estudio y habitación.

Una de las preguntas con baja calificación (entre 3 y 4) fue la calificación de las amenazas. Los expertos, hacen énfasis en que las personas no conocen la calificación posible, probable e inminente; sugieren cambiarla por un sistema numérico de 0 a 3. En los referentes teóricos se sustentan diferentes escalas de valoración, pero en ninguno de los casos una escala numérica de 0 a 3, una de las escalas más usadas es la mencionada, sustentada en estudios previos (Ministerio de la Protección Social, 2007). Es importante especificar que muchas de las preguntas requieren de un encuestador debidamente preparado; esta preparación incluye el manejo conceptual sobre gestión del riesgo de desastres. Lo anterior, implica validez y fiabilidad en los resultados de la investigación (Quispe Parí \& Sánchez Mamani, 2011).

Algunos expertos mencionan la necesidad de quitar la pregunta de reanimación cardiopulmonar, debido a que esta formación se asume en los contenidos de los cursos de primeros auxilios. Es importante analizar, que no existe una correlación entre maniobras de reanimación de reanimación cardiopulmonar (RCP) y los efectos sobre la salud de los desastres. Si bien, es una información importante no se constituye en un factor determinante como vulnerabilidad. Si se han destacado estudios sobre el conocimiento de RCP en la comunidad, pero no están asociados a los desastres (Enríquez et al., 2014; Quintana \& María, 2015). 
Jorge Norvey Álvarez-Ríos, Paula Andrea Aristizábal-Vélez, Diana María Torres-Pavas, Valentina Jurado-Alzate.

Validación de un instrumento para medir la vulnerabilidad en relación con la capacidad

de respuesta de la comunidad ante desastres

\section{Prueba piloto}

En la prueba piloto aplicada, se identificaron dificultades en las preguntas de calificación de la amenaza. Si bien, la terminología es importante, la clasificación de los desastres de acuerdo al origen está sustentada históricamente en "natural y antrópico", no es claro para las personas, el concepto de amenazas de origen antrópico (Salamanca Mazuelo, Quiroga Becerra de la Roca, \& Zamora Auza, 211). Es así, como el encuestador debe recibir buena información para poder aclarar estos conceptos, en su defecto mencionarlo de una manera adecuada sin perder la fiabilidad y sin incidir en la respuesta.

Como medida adicional al instrumento se le agregó la descripción de cada una de las preguntas. Una vez ubicado este componente en el formulario, fue más fácil la aplicación.

\section{Conclusiones}

La validación de instrumentos desde la coherencia, la suficiencia, la claridad y la relevancia a través de los diferentes métodos, ha sido una tendencia en las ciencias; además se ha consolidado como el mejor proceso para identificar si el instrumento sí mide lo que pretende medir (Urrutia Egaña, Barrios Araya, Gutiérrez Núñez, \& Mayorga Camus, 2014).

En gestión del riesgo de desastres se han utilizado múltiples instrumentos, pocos han sido validados para la medición de la vulnerabilidad en relación con la capacidad de respuesta de la comunidad. La presente investigación ha desarrollado una validación por expertos, quienes brindaron una valoración a través de una escala; los resultados demuestran que el instrumento es válido desde el componente cuantitativo, cualitativo y prueba piloto. La prueba de concordancia (Alfa de Cronbach) entre jueces dio mayor a 0,9 , lo cual indica validez interna en relación a relevancia, coherencia, claridad y suficiencia (Cárdenas, Faustino, \& Soler Pons, 2012).

La terminología utilizada en la gestión del riesgo no es tan clara o en su defecto las personas no se han apropiado de ella. Es necesaria la formación y la claridad de conceptos para poder trabajar estos componentes desde lo educativo, lo investigativo y desde la práctica. Lo anterior, sustentado a las múltiples definiciones de riesgo desde las diferentes disciplinas (Echemendía Tocabens, 2011). 
En la suficiencia se menciona la necesidad de quitar la ruta de evacuación, ya que esta solo aplica para conjuntos residenciales. Al realizar el análisis teórico se incluye en el instrumento, debido a que la evidencia demuestra que la ruta no solo es en el interior de la vivienda, sino también en el exterior. De igual forma, se identifica que no es necesario incluir la pregunta de afiliación a seguridad social en salud, debido a que se constituye en una variable distractora.

\section{Referencias}

Aguirre, B. E. (2004). Los desastres en Latinoamérica: vulnerabilidad y resistencia. Revista Mexicana de Sociología, 66(3), 485-510.

Bohórquez, T., \& Enrique, J. (2011). Desarrollo y gestión social del riesgo: ¿una contradicción histórica? Revista de Geografia Norte Grande, (48), 133-157. Recuperado de https://doi.org/10.4067/ S0718-34022011000100008

Cano, V. H. (2011). Aplicación de los sig para la generación de rutas de evacuación en caso de desastres, como ayuda para la planificación urbana: "caso costa oriental del lago de Maracaibo". Revista de La Facultad de Ingeniería Universidad Central de Venezuela, 26(2), 17-26.

Casar, L. P. (2015). Desastres climáticos: anticiparse para reducir el riesgo: Conocer los índices de amenazas y de vulnerabilidad de las regiones minimiza el impacto de los fenómenos meteorológicos, cada vez más frecuentes, que perjudican en mayor medida a los sectores más endebles. Estrategias de resiliencia. RIA. Revista de Investigaciones Agropecuarias, 41(3), 230-234.

Chardon, A. C. (2002). Un enfoque geográfico de la vulnerabilidad en zonas urbanas expuestas a amenazas naturales: el ejemplo andino de Manizales, Colombia. Universidad Nacional de Colombia, Sede Manizales.

Garibay, G. \& Curiel, A. (2002). Percepción del riesgo ambiental en una comunidad universitaria. Investigación en Salud, IV (1), 1-11.

Cid, G. A., Castro, C. P. \& Rugiero de Souza, V. (2012). Percepción del riesgo en relación con capacidades de autoprotección y autogestión, como elementos relevantes en la reducción de la vulnerabilidad en la ciudad de La Serena. Revista INVI, 27(75), 105-142. Recuperado de https://doi.org/10.4067/S0718-83582012000200004 
Jorge Norvey Álvarez-Ríos, Paula Andrea Aristizábal-Vélez, Diana María Torres-Pavas, Valentina Jurado-Alzate.

Validación de un instrumento para medir la vulnerabilidad en relación con la capacidad

de respuesta de la comunidad ante desastres

DeCS Server - List Terms. (n.d.). Retrieved September 1, 2017, Recuperado de http://decs.bvs.br/cgi-bin/wxis1660.exe/decsserver/

Echemendía Tocabens, B. (2011). Definiciones acerca del riesgo y sus implicaciones. Revista Cubana de Higiene y Epidemiología, 49(3), 470-481.

Enríquez, D., Castro, A., Rabasa, C., Capelli, C., Cores Ponte, F., Gutiérrez, S., Szyld, E. (2014). Cursos de reanimación cardiopulmonar básicos para padres de recién nacidos y lactantes. Archivos Argentinos de Pediatría, 112(2), 192-197. Recuperado de https://doi.org/10.5546/aap.2014.192

Fajardo-Maldonado, A. M., Gómez-González, I. M., Corredor-Peñaranda, D. A., Mondragón-Romero, G. M., Ortegón-Rodríguez, J. P., \& Tovar-Cortés, D. L. (2009). Educational Module on School Emergencies. Aquichán, 9(1), 50-61.

Guerrero, R., Gallego, A. I., Becerril-Montekio, V., \& Vásquez, J. (2011). Sistema de salud de Colombia. Salud Pública de México, 53, s144-s155.

Lavin Fueyo, J., \& Berra, S. (2015). Lugares donde niños y niñas realizan actividad física en los barrios periféricos de la ciudad de Córdoba. Salud Colectiva, 11(2), 223-234.

Leonardo Ristori, H. (2011). Respuesta prehospitalaria al evento con múltiples víctimas. Revista Médica Clínica Las Condes, 22(5), 556-565. Recuperado de https://doi.org/10.1016/S0716-8640(11)70466-7

Mardones, M. \& Vidal, C. (2001). La zonificación y evaluación de los riesgos naturales de tipo geomorfológico: un instrumento para la planificación urbana en la ciudad de Concepción. EURE (Santiago), 27(81), 97-122. Recuperado de https://doi.org/10.4067/ S0250-71612001008100006

Medina, F. A. (2013). Identificación de amenazas, gestión de riesgos y propuesta de un plan operacional para enfrentar emergencias o desastres en el Comando General de la Fuerza Aérea Ecuatoriana. Recuperado de http://repositorio.iaen.edu.ec/handle/24000/3817

Ministerio de la Protección Social. (2007). Manual de Planeamiento Hospitalario para Emergencias (Primera). Bogotá, Colombia.

Montenegro-Romero, T., \& Peña-Cortés, F. (2010). Gestión de la emergencia ante eventos de inundación por tsunami en Chile: el caso de Puerto Saavedra. Revista de Geografia Norte Grande, (47), 63-80. Recuperado de https://doi.org/10.4067/S0718-34022010000300004 
Jorge Norvey Álvarez-Ríos, Paula Andrea Aristizábal-Vélez, Diana María Torres-Pavas, Valentina Jurado-Alzate. Validation of an instrument to measure the vulnerability in relation to the community's response capacity front of disasters

Nova, J. A. D.-, Mosqueda, J. S. H.-, \& Tobón, S. T.-. (2016). Juicio De Expertos Para La Validación De Un Instrumento De Medición Del Síndrome De Burnout En La Docencia. Ra Ximhai, 12(6), 327-346.

Pérez, J. E., \& Martínez, Á. C. (2008). Validez de contenido y juicio de expertos: una aproximación a su utilización. Avances en medición, 6(1), 27-36.

Quintana, B., \& María, H. (2015). La Anestesiología-Reanimación en la formación académica del médico de familia. Revista Colombiana de Anestesiología, 43(2), 156-159.

Quispe Parí, D. J., \& Sánchez Mamani, G. (/). Encuestas y entrevistas en investigación científica. Revista de Actualización Clínica Investiga, 490.

Rojas Vilches, O., \& Martínez Reyes, C. (2011). Riesgos naturales: evolución y modelos conceptuales. Revista Universitaria de Geografía, 20(1), 83-116.

Salamanca Mazuelo, L., Quiroga Becerra de la Roca, R., \& Zamora Auza, B. (2011). Ochenta y cinco años de la historia de desastres en Bolivia (1920-2005). Revista Virtual REDESMA, 15.

Sánchez-González, D., \& Egea-Jiménez, C. (2011). Enfoque de vulnerabilidad social para investigar las desventajas socioambientales: $\mathrm{Su}$ aplicación en el estudio de los adultos mayores. Papeles de Población, 17(69), 151-185.

Soares, D., \& Murillo-Licea, D. (2013). Gestión de riesgo de desastres, género y cambio climático. Percepciones sociales en Yucatán, México. Cuadernos de Desarrollo Rural, 10(72), 181-199.

Soler, S. F. \& Soler, L. (2012). Usos del coeficiente alfa de Cronbach en el análisis de instrumentos escritos. Revista Médica Electrónica, 34(1), 01-06.

Thomas Bohórquez, J. E. (2013). Evaluación de la vulnerabilidad social ante amenazas naturales en Manzanillo (Colima). Un aporte de método. Investigaciones Geográficas, Boletín Del Instituto de Geografia, 2013(81), 79-93. Recuperado de https://doi.org/10.14350/rig.36333

Urrutia Egaña, M., Barrios Araya, S., Gutiérrez Núñez, M., \& Mayorga Camus, M. (2014). Métodos óptimos para determinar validez de contenido. Educación Médica Superior, 28(3), 547-558.

Vargas J, J., \& Molina M, G. (2009). Access to health services in six Colombian cities: limitations and consequences. Revista Facultad $\mathrm{Na}$ cional de Salud Pública, 27(2), 121-130. 
Jorge Norvey Álvarez-Ríos, Paula Andrea Aristizábal-Vélez, Diana María Torres-Pavas, Valentina Jurado-Alzate. Validación de un instrumento para medir la vulnerabilidad en relación con la capacidad de respuesta de la comunidad ante desastres

\section{Anexo 1}

\section{Instrumento resultante después de la validación}

\begin{tabular}{|c|c|c|}
\hline DIMENSIÓN & ITEM & OPCIÓN DE RESPUESTA \\
\hline \multirow{6}{*}{$\begin{array}{l}\text { 1. CARACTERÍSTICAS } \\
\text { SOCIODEMOGRÁFICAS } \\
\text { Para el estudio es importante } \\
\text { reconocer la ubicación de la } \\
\text { familia, para esto se utilizará } \\
\text { un muestreo aleatorio por } \\
\text { barrio aplicando un sistema } \\
\text { de georreferenciación. A } \\
\text { continuación, se realizarán } \\
\text { preguntas alrededor de la } \\
\text { ubicación y contexto de la familia. }\end{array}$} & 1.1. Dirección & $\begin{array}{l}\text { Redactar dirección. } \\
\text { Ejemplo calle } 69 \text { No } 34-122\end{array}$ \\
\hline & $\begin{array}{l}\text { 1.2. Barrio } \\
\text { Seleccione el barrio donde } \\
\text { queda ubicada la vivienda }\end{array}$ & $\begin{array}{l}\text { 1) El Campin } \\
\text { 2) El Sol } \\
\text { 3) La Argentina } \\
\text { 4) La Asunción } \\
\text { 5) Lleras } \\
\text { 6) Los Cedros } \\
\text { 7) Santa Helena } \\
\text { 8) San Jorge } \\
\text { 9) Versalles }\end{array}$ \\
\hline & $\begin{array}{l}\text { 1.3. Número de personas que } \\
\text { habitan la vivienda. } \\
\text { Indique el número de personas } \\
\text { por rango de edad que viven } \\
\text { en la vivienda. (Rangos de } \\
\text { edad tomados del Ministerio } \\
\text { de Salud de Colombia) }\end{array}$ & $\begin{array}{l}\text { Cantidad de personas por edad: } \\
\text { 1) } 0-5 \text { años } \\
\text { 2) 6-11 años } \\
\text { 3) } 12-18 \text { años } \\
\text { 4) } 14-26 \text { años } \\
\text { 5) } 27-29 \text { años } \\
\text { 6) } 60 \text { años y más }\end{array}$ \\
\hline & $\begin{array}{l}\text { 1.4. Antigüedad en el barrio } \\
\text { Número de años que lleva } \\
\text { viviendo la familia en el } \\
\text { barrio }\end{array}$ & $\begin{array}{l}\text { 1) Entre } 1 \text { y } 5 \text { años } \\
\text { 2) Entre } 6 \text { y } 10 \text { años } \\
\text { 3) Entre } 11 \text { y } 15 \text { años } \\
\text { 4) Más de } 15 \text { años }\end{array}$ \\
\hline & $\begin{array}{l}\text { 1.5. Que lo motivo a vivir en } \\
\text { este sector } \\
\text { Descripción del motivo por } \\
\text { el cual decidieron vivir en } \\
\text { el barrio }\end{array}$ & $\begin{array}{l}\text { Descripción del motivo por el cual } \\
\text { decidieron vivir en el barrio }\end{array}$ \\
\hline & $\begin{array}{l}\text { 1.6. Pertenece usted o algún } \\
\text { miembro de su familia a } \\
\text { alguna organización como: }\end{array}$ & $\begin{array}{l}\text { 1) Junta de Acción Local (JAL) } \\
\text { 2) Junta de Acción Comunal (JAC) } \\
\text { 3) Bomberos } \\
\text { 4) Cruz Roja Colombiana } \\
\text { 5) Defensa Civil Colombiana } \\
\text { 6) Brigada de emergencias escolar o } \\
\text { empresarial } \\
\text { 7) Otro }\end{array}$ \\
\hline
\end{tabular}


Jorge Norvey Álvarez-Ríos, Paula Andrea Aristizábal-Vélez, Diana María Torres-Pavas, Valentina Jurado-Alzate. Validation of an instrument to measure the vulnerability in relation to the community's response capacity front of disasters

\begin{tabular}{|c|c|c|}
\hline DIMENSIÓN & ITEM & OPCIÓN DE RESPUESTA \\
\hline $\begin{array}{l}\text { 1. CARACTERÍSTICAS } \\
\text { SOCIODEMOGRÁFICAS } \\
\text { Para el estudio es importante } \\
\text { reconocer la ubicación de la } \\
\text { familia, para esto se utilizará } \\
\text { un muestreo aleatorio por } \\
\text { barrio aplicando un sistema } \\
\text { de georreferenciación. A } \\
\text { continuación, se realizarán } \\
\text { preguntas alrededor de la } \\
\text { ubicación y contexto de la familia. }\end{array}$ & $\begin{array}{l}\text { 1.7. Máximo nivel de } \\
\text { escolaridad logrado por } \\
\text { algún integrante de la } \\
\text { vivienda }\end{array}$ & $\begin{array}{l}\text { 1) Ninguno } \\
\text { 2) Primaria } \\
\text { 3) Secundaria } \\
\text { 4) Técnico } \\
\text { 5) Tecnológico } \\
\text { 6) Universidad } \\
\text { 7) Especialización } \\
\text { 8) Maestría } \\
\text { 9) Doctorado } \\
\text { 10) Otro } \\
\end{array}$ \\
\hline & 2.1. Sexo & $\begin{array}{l}\text { 1) Masculino } \\
\text { 2) Femenino }\end{array}$ \\
\hline & 2.2. Ocupación & Respuesta corta \\
\hline $\begin{array}{l}\text { 2. INFORMACIÓN DE LA } \\
\text { PERSONA ENCUESTADA }\end{array}$ & $\begin{array}{l}\text { 2.3. Edad } \\
\text { Edad de quien responde la } \\
\text { encuesta }\end{array}$ & $\begin{array}{l}\text { 1) Entre } 18 \text { y } 26 \text { años } \\
\text { 2) Entre } 27 \text { y } 59 \text { años } \\
\text { 3) } 60 \text { o más años }\end{array}$ \\
\hline $\begin{array}{l}\text { Información general de la persona } \\
\text { quien atiende al encuestador. Esta } \\
\text { información es especifica de la } \\
\text { persona que responde la encuesta, } \\
\text { no incluye información de las demás } \\
\text { personas que viven en el hogar. }\end{array}$ & $\begin{array}{l}\text { 2.4. Máximo nivel de } \\
\text { estudios } \\
\text { Máximo nivel de formación } \\
\text { de quien responde la } \\
\text { encuesta }\end{array}$ & $\begin{array}{l}\text { 1) Ninguno } \\
\text { 2) Primaria } \\
\text { 3) Secundaria } \\
\text { 4) Técnico } \\
\text { 5) Tecnológico } \\
\text { 6) Universidad } \\
\text { 7) Especialización } \\
\text { 8) Maestría } \\
\text { 9) Doctorado } \\
\text { 10) Otro }\end{array}$ \\
\hline $\begin{array}{l}\text { 3. CARACTERÍSTICAS } \\
\text { ECONÓMICAS } \\
\text { La información aquí suministrada } \\
\text { permitirá evaluar la posible }\end{array}$ & $\begin{array}{l}\text { 3.1. Ingresos mensuales } \\
\text { familiares } \\
\text { Indique número de Salario } \\
\text { Minimo Mensual Legal } \\
\text { Vigente (SMMLV) }\end{array}$ & $\begin{array}{l}\text { 1) Menor a } 1 \text { SMMLV } \\
\text { 2) } 1 \text { SMMLV } \\
\text { 3) Entre } 1 \text { y } 2 \text { SMMLV } \\
\text { 4) Entre } 2 \text { y } 3 \text { SMMLV } \\
\text { 5) } 3 \text { o más SMMLV }\end{array}$ \\
\hline $\begin{array}{l}\text { capacidad de recuperación de la } \\
\text { familia frente a un evento adverso } \\
\text { o de instaurar medidas fisicas } \\
\text { correctivas. Este apartado incluye } \\
\text { información alrededor de los } \\
\text { ingresos mensuales en la vivienda. }\end{array}$ & 3.2. Estrato socioeconómico & $\begin{array}{l}\text { 1) } 1 \\
\text { 2) } 2 \\
\text { 3) } 3 \\
\text { 4) } 4 \\
\text { 5) } 5 \\
\text { 6) } 6\end{array}$ \\
\hline
\end{tabular}


Jorge Norvey Álvarez-Ríos, Paula Andrea Aristizábal-Vélez, Diana María Torres-Pavas, Valentina Jurado-Alzate. Validación de un instrumento para medir la vulnerabilidad en relación con la capacidad de respuesta de la comunidad ante desastres

\begin{tabular}{|c|c|c|}
\hline DIMENSIÓN & ITEM & OPCIÓN DE RESPUESTA \\
\hline \multirow{11}{*}{$\begin{array}{l}\text { 4. CARACTERÍSTICAS DE LA } \\
\text { VIVIENDA } \\
\text { Este apartado permite evidenciar } \\
\text { la vulnerabilidad estructural. } \\
\text { En este componente se deben } \\
\text { responder las preguntas asociadas } \\
\text { a las características de la vivienda } \\
\text { donde se está aplicando el } \\
\text { instrumento. }\end{array}$} & 4.1. Su vivienda es: & $\begin{array}{l}\text { 1) Propia } \\
\text { 2) Arrendada } \\
\text { 3) Familiar }\end{array}$ \\
\hline & 4.2. Tipo de vivienda: & $\begin{array}{l}\text { 1) Casa } \\
\text { 2) Apartamento } \\
\text { 3) Otro }\end{array}$ \\
\hline & 4.3. Número de pisos: & $\begin{array}{l}\text { 1) Uno } \\
\text { 2) Dos } \\
\text { 3) Tres } \\
\text { 4) Otro }\end{array}$ \\
\hline & $\begin{array}{l}\text { 4.4. Tipo de construcción: } \\
\text { Indique el material principal } \\
\text { por el cual está construida } \\
\text { su vivienda }\end{array}$ & $\begin{array}{l}\text { 1) Concreto } \\
\text { 2) Ladrillo } \\
\text { 3) Bahareque } \\
\text { 4) Madera } \\
\text { 5) Prefabricada } \\
\text { 6) Otro }\end{array}$ \\
\hline & $\begin{array}{l}4.5 . \text { ¿Quién construyó la } \\
\text { vivienda? } \\
\text { Indique quien se } \\
\text { responsabilizó de la } \\
\text { construcción de la vivienda }\end{array}$ & $\begin{array}{l}\text { 1) Constructora } \\
\text { 2) Usted mismo } \\
\text { 3) No sabe }\end{array}$ \\
\hline & $\begin{array}{l}\text { 4.6. Área construida en } \\
\text { metros cuadrados } \\
\text { Indique en metros } \\
\text { cuadrados (Largo x ancho) } \\
\text { aproximadamente }\end{array}$ & Metros cuadrados aprox. \\
\hline & $\begin{array}{l}\text { 4.7. ¿La vivienda está } \\
\text { totalmente terminada? }\end{array}$ & $\begin{array}{l}\text { 1) } \mathrm{Si} \\
\text { 2) } \mathrm{No}\end{array}$ \\
\hline & $\begin{array}{l}\text { 4.8. ¿La vivienda fue } \\
\text { construida hace cuantos años? }\end{array}$ & $\begin{array}{l}\text { Poner la cantidad en años o en su } \\
\text { defecto no sabe }\end{array}$ \\
\hline & $\begin{array}{l}\text { 4.9. ¿Se ha realizado } \\
\text { mantenimiento a la vivienda? } \\
\text { Incluye mantenimiento de } \\
\text { canales, techos, paredes, } \\
\text { entre otros. }\end{array}$ & \\
\hline & $\begin{array}{l}\text { 4.10. ¿En la vivienda se } \\
\text { ha incorporado normas de } \\
\text { sismo resistencia? }\end{array}$ & $\begin{array}{l}\text { 1) } \mathrm{Si} \\
\text { 2) No } \\
\text { 3) No sabe }\end{array}$ \\
\hline & 4.11. Acerca del uso del suelo: & $\begin{array}{l}\text { 1) Solo vivienda } \\
\text { 2) Vivienda y comercio } \\
\text { 3) Vivienda e industria } \\
\text { 4) Solo comercio y/o industria }\end{array}$ \\
\hline
\end{tabular}


Jorge Norvey Álvarez-Ríos, Paula Andrea Aristizábal-Vélez, Diana María Torres-Pavas, Valentina Jurado-Alzate. Validation of an instrument to measure the vulnerability in relation to the community's response capacity front of disasters

\begin{tabular}{|c|c|c|}
\hline DIMENSIÓN & ITEM & OPCIÓN DE RESPUESTA \\
\hline \multirow{2}{*}{$\begin{array}{l}\text { 4. CARACTERÍSTICAS DE LA } \\
\text { VIVIENDA } \\
\text { Este apartado permite evidenciar } \\
\text { la vulnerabilidad estructural. } \\
\text { En este componente se deben } \\
\text { responder las preguntas asociadas } \\
\text { a las características de la vivienda } \\
\text { donde se está aplicando el } \\
\text { instrumento. }\end{array}$} & $\begin{array}{l}\text { 4.12. La vivienda cuenta con } \\
\text { servicios de: }\end{array}$ & $\begin{array}{l}\text { Varias opciones: } \\
\text { 1) Energía eléctrica } \\
\text { 2) Acueducto } \\
\text { 3) Alcantarillado } \\
\text { 4) Teléfono fijo } \\
\text { 5) Teléfono celular } \\
\text { 6) Cable } \\
\text { 7) Internet } \\
\text { 8) Gas por cilindro } \\
\text { 9) Gas domiciliario }\end{array}$ \\
\hline & $\begin{array}{l}\text { 4.13. El barrio cuenta con } \\
\text { servicio de: }\end{array}$ & $\begin{array}{l}\text { 1) Recolección de basuras } \\
\text { 2) Transporte público } \\
\text { 3) Hospitales, centros de salud o clínicas } \\
\text { 4) Supermercados } \\
\text { 5) Escuelas y colegios } \\
\text { 6) Vigilancia } \\
\text { 8) CAI }\end{array}$ \\
\hline \multirow[b]{2}{*}{$\begin{array}{l}\text { 5. AMENAZAS NATURALES } \\
\text { El conocimiento de las amenazas } \\
\text { es uno de los elementos } \\
\text { esenciales del estudio, el análisis } \\
\text { correlacional entre las amenazas } \\
\text { percibidas y las identificadas por } \\
\text { las entidades es determinante. } \\
\text { En este componente se realizan } \\
\text { preguntas alrededor de las } \\
\text { amenazas de origen natural que } \\
\text { la persona encuestada identifica } \\
\text { alrededor o en su vivienda. }\end{array}$} & $\begin{array}{l}\text { 5.1. ¿Conoce usted algún } \\
\text { evento de origen natural } \\
\text { que ponga en amenaza su } \\
\text { vivienda? } \\
\text { Eventos como } \\
\text { desprendimiento de tierra, } \\
\text { sismos, entre otros }\end{array}$ & $\begin{array}{l}\text { 1) } \mathrm{Si} \\
\text { 2) } \mathrm{No}\end{array}$ \\
\hline & $\begin{array}{l}\text { 5.2. Califique las amenazas } \\
\text { según su probabilidad de } \\
\text { ocurrencia en su vivienda } \\
\text { Esta pregunta se responde } \\
\text { según los siguientes } \\
\text { criterios: Inminente: el } \\
\text { evento ha ocurrido y en } \\
\text { cualquier momento vuelve a } \\
\text { ocurrir; probable: el evento } \\
\text { ha ocurrido anteriormente y } \\
\text { se puede volver a presentar; } \\
\text { posible: el evento no ha } \\
\text { ocurrido pero se podría } \\
\text { presentar. No es probable } \\
\text { que ocurra: para la persona } \\
\text { que responde no es posible } \\
\text { ni probable que ocurra un } \\
\text { evento como estos. }\end{array}$ & $\begin{array}{l}\text { Amenazas: } \\
\text { 1) Deslizamiento } \\
\text { 2) Sismo } \\
\text { 3) Erupción Volcánica } \\
\text { 4) Inundación } \\
\text { 5) Vendaval } \\
\text { 6) Otra } \\
\text { Calificación: } \\
\text { 1) Inminente } \\
\text { 2) Probable } \\
\text { 3) Posible } \\
\text { 4) No es posible que ocurra }\end{array}$ \\
\hline
\end{tabular}


Jorge Norvey Álvarez-Ríos, Paula Andrea Aristizábal-Vélez, Diana María Torres-Pavas, Valentina Jurado-Alzate. Validación de un instrumento para medir la vulnerabilidad en relación con la capacidad de respuesta de la comunidad ante desastres

\begin{tabular}{|c|c|c|}
\hline DIMENSIÓN & ITEM & OPCIÓN DE RESPUESTA \\
\hline \multirow{3}{*}{$\begin{array}{l}\text { 5. AMENAZAS NATURALES } \\
\text { El conocimiento de las amenazas } \\
\text { es uno de los elementos } \\
\text { esenciales del estudio, el análisis } \\
\text { correlacional entre las amenazas } \\
\text { percibidas y las identificadas por } \\
\text { las entidades es determinante. } \\
\text { En este componente se realizan } \\
\text { preguntas alrededor de las } \\
\text { amenazas de origen natural que } \\
\text { la persona encuestada identifica } \\
\text { alrededor o en su vivienda. }\end{array}$} & $\begin{array}{l}\text { 5.3. Indique los fenómenos } \\
\text { de origen naturales que han } \\
\text { ocurrido en el barrio } \\
\text { Busca calificar la ocurrencia } \\
\text { de los eventos }\end{array}$ & $\begin{array}{l}\text { 1) Deslizamiento } \\
\text { 2) Sismo } \\
\text { 3) Erupción Volcánica } \\
\text { 4) Inundación } \\
\text { 5) Vendaval } \\
\text { 6) Ninguno } \\
\text { 7) Otro }\end{array}$ \\
\hline & $\begin{array}{l}\text { 5.4. ¿Se siente en peligro por } \\
\text { esta amenaza? }\end{array}$ & $\begin{array}{l}\text { 1) } \mathrm{Si} \\
\text { 2) No } \\
\text { Si la respuesta anterior fue afirmativa } \\
\text { ¿Cuánto?: } \\
\text { 1) Mucho } \\
\text { 2) Medianamente } \\
\text { 3) Nada }\end{array}$ \\
\hline & $\begin{array}{l}\text { 5.5. ¿Sabe cómo esos } \\
\text { eventos pueden afectar la } \\
\text { vivienda, a usted y a su } \\
\text { familia? }\end{array}$ & Descripción \\
\hline \multirow[b]{2}{*}{$\begin{array}{l}\text { 6. AMENAZAS ANTRÓPICAS } \\
\text { El conocimiento de las amenazas } \\
\text { es uno de los elementos } \\
\text { esenciales del estudio, el análisis } \\
\text { correlacional entre las amenazas } \\
\text { percibidas y las identificadas por } \\
\text { las entidades, es determinante. } \\
\text { En este componente se realizan } \\
\text { preguntas alrededor de las } \\
\text { amenazas de origen antrópico que } \\
\text { la persona encuestada identifica } \\
\text { alrededor o en su vivienda. }\end{array}$} & \multirow[b]{2}{*}{$\begin{array}{l}\text { 6.1. ¿Conoce usted } \\
\text { algún evento de origen } \\
\text { antrópico que ponga en } \\
\text { amenaza su vivienda? } \\
\text { Esta pregunta se responde } \\
\text { según los siguientes } \\
\text { criterios: Inminente: el } \\
\text { evento ha ocurrido y en } \\
\text { cualquier momento vuelve a } \\
\text { ocurrir; probable: el evento } \\
\text { ha ocurrido anteriormente y } \\
\text { se puede volver a presentar; } \\
\text { posible: el evento no ha } \\
\text { ocurrido, pero se podria } \\
\text { presentar. No es probable } \\
\text { que ocurra: para la persona } \\
\text { que responde no es posible } \\
\text { ni probable que ocurra un } \\
\text { evento como estos. }\end{array}$} & 1) $\mathrm{Si}$ \\
\hline & & 2) $\mathrm{No}$ \\
\hline
\end{tabular}


Jorge Norvey Álvarez-Ríos, Paula Andrea Aristizábal-Vélez, Diana María Torres-Pavas, Valentina Jurado-Alzate. Validation of an instrument to measure the vulnerability in relation to the community's response capacity front of disasters

\begin{tabular}{|c|c|c|}
\hline DIMENSIÓN & ITEM & OPCIÓN DE RESPUESTA \\
\hline \multirow{4}{*}{$\begin{array}{l}\text { 6. AMENAZAS ANTRÓPICAS } \\
\text { El conocimiento de las amenazas } \\
\text { es uno de los elementos } \\
\text { esenciales del estudio, el análisis } \\
\text { correlacional entre las amenazas } \\
\text { percibidas y las identificadas por } \\
\text { las entidades, es determinante. } \\
\text { En este componente se realizan } \\
\text { preguntas alrededor de las } \\
\text { amenazas de origen antrópico que } \\
\text { la persona encuestada identifica } \\
\text { alrededor o en su vivienda. }\end{array}$} & $\begin{array}{l}\text { 6.2. Califique las amenazas } \\
\text { según su probabilidad de } \\
\text { ocurrencia en su vivienda }\end{array}$ & $\begin{array}{l}\text { Amenazas: } \\
\text { 1) Explosión } \\
\text { 2) Atentados terroristas } \\
\text { 3) Incendio } \\
\text { 4) Accidente químico } \\
\text { 5) Accidente de tránsito } \\
\text { 6) Otro } \\
\text { Calificación: } \\
\text { 1) Inminente } \\
\text { 2) Probable } \\
\text { 3) Posible } \\
\text { 4) No es posible que ocurra }\end{array}$ \\
\hline & $\begin{array}{l}\text { 6.3. Indique los fenómenos } \\
\text { de origen antrópico que han } \\
\text { ocurrido en el barrio }\end{array}$ & $\begin{array}{l}\text { 1) Explosión } \\
\text { 2) Atentados terroristas } \\
\text { 3) Incendio } \\
\text { 4) Accidente químico } \\
\text { 5) Accidente de tránsito } \\
\text { 6) Otro } \\
\text { 7) Ninguno }\end{array}$ \\
\hline & $\begin{array}{l}\text { 6.4. ¿Se siente en peligro por } \\
\text { esta amenaza? }\end{array}$ & $\begin{array}{l}\text { 1) } \mathrm{Si} \\
\text { 2) No } \\
\text { Si la anterior respuesta fue afirmativa } \\
\text { ¿Cuánto?: } \\
\text { 1) Mucho } \\
\text { 2) Medianamente } \\
\text { 3) Nada }\end{array}$ \\
\hline & $\begin{array}{l}\text { 6.5. ¿Sabe cómo esos eventos } \\
\text { pueden afectar la vivienda, a } \\
\text { usted y a su familia? }\end{array}$ & $\begin{array}{l}\text { 1) } \mathrm{Si} \\
\text { 2) } \mathrm{No}\end{array}$ \\
\hline $\begin{array}{l}\text { 7. ORGANIZACIÓN ANTE LAS } \\
\text { AMENAZAS } \\
\text { Este componente busca evaluar } \\
\text { la organización de la familia para } \\
\text { disminuir la vulnerabilidad frente } \\
\text { a los eventos adversos. En este } \\
\text { componente el encuestado expresa }\end{array}$ & $\begin{array}{l}\text { 7.1. ¿Su familia sabe que es } \\
\text { un plan familiar de gestión } \\
\text { del riesgo de desastres? } \\
\text { La persona expresa si } \\
\text { cuenta con un documento } \\
\text { o acuerdos previos de la } \\
\text { familia de organización } \\
\text { para enfrentar una situación } \\
\text { adversa de desastre. }\end{array}$ & $\begin{array}{l}\text { 1) } \mathrm{Si} \\
\text { 2) } \mathrm{No}\end{array}$ \\
\hline $\begin{array}{l}\text { la organización de la familia frente a } \\
\text { los eventos naturales y antrópicos. }\end{array}$ & $\begin{array}{l}\text { 7.2. ¿Su familia cuenta con } \\
\text { un plan de gestión del riesgo } \\
\text { de desastres? }\end{array}$ & $\begin{array}{l}\text { 1) } \mathrm{Si} \\
\text { 2) } \mathrm{No}\end{array}$ \\
\hline
\end{tabular}


Jorge Norvey Álvarez-Ríos, Paula Andrea Aristizábal-Vélez, Diana María Torres-Pavas, Valentina Jurado-Alzate. Validación de un instrumento para medir la vulnerabilidad en relación con la capacidad de respuesta de la comunidad ante desastres

\begin{tabular}{|c|c|c|}
\hline DIMENSIÓN & ITEM & OPCIÓN DE RESPUESTA \\
\hline \multirow[b]{2}{*}{$\begin{array}{l}\text { 7. ORGANIZACIÓN ANTE LAS } \\
\text { AMENAZAS } \\
\text { Este componente busca evaluar } \\
\text { la organización de la familia para } \\
\text { disminuir la vulnerabilidad frente } \\
\text { a los eventos adversos. En este } \\
\text { componente el encuestado expresa } \\
\text { la organización de la familia frente a } \\
\text { los eventos naturales y antrópicos. }\end{array}$} & $\begin{array}{l}\text { 7.3. ¿La vivienda cuenta con } \\
\text { rutas de evacuación? } \\
\text { Estas rutas son vias } \\
\text { de salida en caso de la } \\
\text { presencia de un evento } \\
\text { adverso (sismo, incendio, } \\
\text { deslizamiento, entre } \\
\text { otros) Describe acuerdos } \\
\text { previos o rutas señalizadas } \\
\text { establecidas por la familia. }\end{array}$ & $\begin{array}{l}\text { 1) } \mathrm{Si} \\
\text { 2) } \mathrm{No}\end{array}$ \\
\hline & $\begin{array}{l}\text { 7.4. Si ocurre un evento } \\
\text { adverso que requiera la } \\
\text { evacuación ¿Su familia cuenta } \\
\text { con un punto de reunión } \\
\text { previamente definido? } \\
\text { El punto de encuentro son } \\
\text { áreas libres de riesgo que } \\
\text { se utilizan para llegar } \\
\text { alli en caso de un evento } \\
\text { adverso que puede afectar } \\
\text { la vivienda o la comunidad. } \\
\text { Este punto de encuentro } \\
\text { puede ser comunitario o } \\
\text { en su defecto en un lugar } \\
\text { establecido en la ciudad } \\
\text { (ejemplo: el punto de } \\
\text { encuentro puede ser un } \\
\text { hospital, casa de algún } \\
\text { familiar, entre otros) }\end{array}$ & $\begin{array}{l}\text { 1) } \mathrm{Si} \\
\text { 2) } \mathrm{No}\end{array}$ \\
\hline \multirow{10}{*}{$\begin{array}{l}\text { 8. PREPARACIÓN ANTE LAS } \\
\text { AMENAZAS } \\
\text { La preparación identifica la } \\
\text { formación y dotación para atender } \\
\text { una situación adversa. En este } \\
\text { componente el encuestado expresa } \\
\text { la preparación de la familia } \\
\text { frente a los eventos naturales y } \\
\text { antrópicos. }\end{array}$} & \multirow{2}{*}{$\begin{array}{l}\text { 8.1. ¿Usted o algún } \\
\text { integrante de la familia } \\
\text { ha recibido formación } \\
\text { en gestión del riesgo de } \\
\text { desastres? }\end{array}$} & 1) $\mathrm{Si}$ \\
\hline & & 2) No \\
\hline & \multirow{2}{*}{$\begin{array}{l}\text { 8.2. ¿Usted o algún } \\
\text { integrante de la familia } \\
\text { conoce el plan de gestión } \\
\text { del riesgo de desastres de } \\
\text { Manizales? }\end{array}$} & 1) $\mathrm{Si}$ \\
\hline & & 2) No \\
\hline & \multirow{3}{*}{$\begin{array}{l}\text { 8.3. ¿Conoce usted si la } \\
\text { comunidad cuenta con un } \\
\text { plan de gestión del riesgo de } \\
\text { desastres? }\end{array}$} & 1) $\mathrm{Si}$ \\
\hline & & 2) $\mathrm{No}$ \\
\hline & & 3) No existe \\
\hline & \multirow{3}{*}{$\begin{array}{l}\text { 8.4. ¿Conoce usted si la } \\
\text { comunidad cuenta con un } \\
\text { sistema de alarma frente a } \\
\text { eventos de origen natural o } \\
\text { antrópico? }\end{array}$} & 1) $\mathrm{Si}$ \\
\hline & & 2) $\mathrm{No}$ \\
\hline & & 3) No existe sistema \\
\hline
\end{tabular}


Jorge Norvey Álvarez-Ríos, Paula Andrea Aristizábal-Vélez, Diana María Torres-Pavas, Valentina Jurado-Alzate. Validation of an instrument to measure the vulnerability in relation to the community's response capacity front of disasters

\begin{tabular}{|c|c|c|}
\hline DIMENSIÓN & ITEM & OPCIÓN DE RESPUESTA \\
\hline \multirow{7}{*}{$\begin{array}{l}\text { 8. PREPARACIÓN ANTE LAS } \\
\text { AMENAZAS } \\
\text { La preparación identifica la } \\
\text { formación y dotación para atender } \\
\text { una situación adversa. En este } \\
\text { componente el encuestado expresa } \\
\text { la preparación de la familia } \\
\text { frente a los eventos naturales y } \\
\text { antrópicos. }\end{array}$} & $\begin{array}{l}8.5 \text { ¿Su familia cuenta con } \\
\text { un botiquín debidamente } \\
\text { dotado? }\end{array}$ & $\begin{array}{l}\text { 1) } \mathrm{Si} \\
\text { 2) } \mathrm{No}\end{array}$ \\
\hline & $\begin{array}{l}\text { 8.6. ¿Ha recibido usted o } \\
\text { algún integrante de la familia } \\
\text { formación en primeros } \\
\text { auxilios? }\end{array}$ & $\begin{array}{l}\text { 1) } \mathrm{Si} \\
\text { 2) } \mathrm{No}\end{array}$ \\
\hline & $\begin{array}{l}\text { 8.7. ¿Conoce el número de } \\
\text { emergencias de la ciudad de } \\
\text { Manizales? }\end{array}$ & $\begin{array}{l}\text { 1) } \mathrm{Si} \\
\text { 2) } \mathrm{No}\end{array}$ \\
\hline & $\begin{array}{l}\text { 8.8. ¿Cuenta su hogar con un } \\
\text { extintor? }\end{array}$ & $\begin{array}{l}\text { 1) } \mathrm{Si} \\
\text { 2) } \mathrm{No}\end{array}$ \\
\hline & $\begin{array}{l}\text { 8.9. ¿Ha recibido usted } \\
\text { o algún integrante de la } \\
\text { familia formación en manejo } \\
\text { de extintores? }\end{array}$ & $\begin{array}{l}\text { 1) } \mathrm{Si} \\
\text { 2) } \mathrm{No}\end{array}$ \\
\hline & $\begin{array}{l}\text { 8.10. Si ha recibido } \\
\text { formación de alguno de los } \\
\text { temas anteriores ¿Por qué } \\
\text { medio lo recibido? }\end{array}$ & $\begin{array}{l}\text { 1) Periódico } \\
\text { 2) Radio } \\
\text { 3) Televisión } \\
\text { 4) Volantes } \\
\text { 5) Curso de formación corta } \\
\text { 6) Reuniones } \\
\text { 7) No ha recibido formación } \\
\text { 8) Otro }\end{array}$ \\
\hline & $\begin{array}{l}\text { 8.11. ¿Le gustaría recibir } \\
\text { información alrededor de la } \\
\text { preparación frente a eventos } \\
\text { naturales y antrópicos? }\end{array}$ & $\begin{array}{l}\text { 1) } \mathrm{Si} \\
\text { 2) No } \\
\text { Si la respuesta anterior fue afirmativa, } \\
\text { ¿Por qué medio le gustaría recibir } \\
\text { información? } \\
\text { 1) Periódico } \\
\text { 2) Radio } \\
\text { 3) Televisión } \\
\text { 4) Volantes } \\
\text { 5) Curso de formación corta } \\
\text { 6) Reuniones } \\
\text { 7) Otro }\end{array}$ \\
\hline
\end{tabular}

